Fernández F, Burgos R, Kennedy ML, Campuzano-Bublitz

MA. Efecto del consumo de miel de abeja sobre la glicemia

ARTÍCULO ORIGINAL en ratones normo e hiperglucémicos por aloxano. Rev. Soc. ORIGINAL ARTICLE cient. Parag. 2021;26(1):91-99.

https://doi.org/10.32480/rscp.2021.26.1.91

Recibido: 14/04/2021. Aceptado: 24/05/2021.

\title{
Efecto del consumo de miel de abeja sobre la glicemia en ratones normo e hiperglucémicos por aloxano
}

\section{Effect of consumption of honey on glycemia in normal and hyperglycemic mice due to alloxane}

\author{
Fátima Fernández ${ }^{1}$ (D) , Rodrigo Burgos ${ }^{1}$ (D), María Luisa Kennedy ${ }^{1}$ \\ Miguel A. Campuzano-Bublitz ${ }^{1^{*}}$ \\ ${ }^{1}$ Universidad Nacional de Asunción, Facultad de Ciencias Químicas, \\ Departamento de Farmacología. San Lorenzo, Paraguay.
}

Autor correspondiente: mbublitz@qui.una.py

Resumen: La Diabetes Mellitus (DM) es una patología crónica de origen metabólico, que aparece debido a que el páncreas no produce la cantidad de insulina que el cuerpo humano necesita, o bien de una calidad inferior. En este trabajo pretendimos definir el efecto del consumo de la miel de abeja sobre la glucemia en ratones macho sanos e hiperglucémicos inducidos a diabetes por aloxano. Para ello se emplearon cuatro grupos de seis ratones albinos suizos machos cada uno. normoglucémicos con dieta regular tratados con agua; normoglucémicos con dieta + miel de abeja; hiperglicémicos con dieta regular tratados con agua y finalmente, hiperglicémicos con aloxano con dieta + miel. El tratamiento se realizó por tres días. Los resultados indicaron que los animales hipeglucémicos que recibieron la miel por tres días mostraron un perfil favorable en la glicemia, ya que se observó un descenso significativo del nivel de azúcar, en este grupo luego de 2 y 4 horas del consumo de la miel. La adición de miel de abeja en el grupo de animales hiperglicémicos con dieta controlada contribuye a alcanzar glicemias significativamente menores que en el grupo de hiperglicémicos que no reciben miel.

Palabras clave: ratón albino suizo, miel de abeja, aloxano, hiperglucemia. 
Abstract: Diabetes Mellitus is a chronic pathology of metabolic origin, which appears due to the fact that the pancreas does not produce the amount of insulin that the human body needs, or of a lower quality. In this work we tried to define the effect of the consumption of honey on the glycemia in healthy and hyperglycemic male mice induced to diabetes by alloxane. For this, four groups of six male Swiss albino mice each were used. normoglycemic with regular diet treated with water; normoglycemic with diet + honey; hyperglycemic with regular diet treated with water and finally, hyperglycemic with alloxane with diet + honey. The treatment lasted for three days. The results indicated that the hypeglycemic animals that received honey for three days showed a favorable glycemic profile, since a significant decrease in sugar level was observed in this group after 2 and 4 hours of consumption of honey. The addition of honey in the group of hyperglycemic animals with a controlled diet contributes to achieving significantly lower glycaemia than in the group of hyperglycemic animals that do not receive honey.

Keywords: Swiss albino mouse, honey, alloxane, hyperglycemia.

\section{INTRODUCCIÓN}

La Diabetes Mellitus (DM) es una crónica, que cursa con hiperglicemia y aparece debido a que el páncreas no produce la cantidad de insulina que el cuerpo humano necesita(1). La insulina, es una hormona anabólica, es decir promueve reservas de energía, es la principal sustancia responsable del mantenimiento de los valores adecuados de glucosa en sangre ${ }^{(2)}$. Permite que la misma sea transportada al interior de las células, de modo que éstas produzcan energía o almacenen la glucosa hasta que su utilización sea necesaria. Cuando falla, origina un aumento excesivo de la glucosa en sangre llamada hiperglucemia. La diabetes se clasifica en: diabetes tipo 1 que depende de la insulina exógena, diabetes tipo 2 que se caracteriza por resistencia a la insulina y deficiencia relativa de esta hormona, y la diabetes gestacional que es cualquier grado de intolerancia a la glucosa que comienza o se reconoce por primera vez durante el embarazo y culmina con la misma(2).

En Paraguay, la diabetes afecta al 6,5\% de la población, unas 400.000 personas aproximadamente. El $85 \%$ de los afectados presentan el tipo 2 , y el restante $15 \%$ presentan el tipo 1 . El momento de aparición de la enfermedad, así como las causas y los síntomas que presentan los pacientes dependen del tipo de DM que se trate. Las posibilidades de 
contraer la enfermedad aumentan a medida que una persona se hace mayor, de modo que por encima de los 55 años la padece alrededor del $15 \%$ de las personas. Entre las causas que conducen a la diabetes se encuentran la obesidad, sedentarismo, edad avanzada, factores genéticos, infección viral, consumo excesivo de calorías, en donde la alimentación aporta alta cantidad de hidratos de carbono simple(4).

La fructosa o levulosa es una forma de azúcar encontrada en las frutas y en la miel, se utiliza como edulcorante para los diabéticos ya que no requiere de insulina para ser metabolizada. A diferencia del azúcar refinado o de mesa que se absorbe instantáneamente produciendo picos de subida y bajada rápidos de la glucemia, la fructosa es metabolizada y almacenada, en parte, por el hígado en forma de glucógeno como reserva para cuando se necesite hacer un esfuerzo. La fructosa produce escasos efectos en el nivel de glucemia y no estimula la secreción de insulina. Resulta ideal en dietas que necesiten tener lo más equilibrado posible los niveles de insulina como diabéticos, deportistas y sobre todo personas que quieren adelgazar(5).

Existen escasos estudios sobre la búsqueda de un efecto beneficioso de la miel de abeja sobre la diabetes. Teniendo en cuenta que en Paraguay no existen estudios sobre dicho efecto, y que la miel de abeja es consumida habitualmente por la población, podría constituir un edulcorante alternativo o una terapia coadyuvante por demás innovador, a la dieta controlada; por lo tanto, nos propusimos realizar el presente trabajo a fin de establecer el efecto del consumo de la miel de abeja en los niveles de glucosa en sangre en ratones albinos suizos machos inducidos a diabetes experimental mediante aloxano.

\section{MATERIALES Y MÉTODOS}

\subsection{Materiales y reactivos}

Glucómetro y tiras reactivas "codefree", (proveídas por el Programa Nacional de Diabetes), báscula ASPEN, cánula para administración oral, jeringas para insulina (Mediley Paraguay S.A.), monohidarato de aloxano de Sigma-Aldrich, balanceado comercial "Supermix roedores", miel de abeja (Ju'i Importación - Exportación para su marca Don Enrique), producida en Cerrito, Ñeembucú. 


\subsection{Animales de experimentación}

Fueron empleados ratones albinos suizos machos de 30 a 40 gramos de peso aproximadamente, criados y mantenidos en el bioterio del Departamento de Farmacología de la Facultad de Ciencias Químicas, UNA, aclimatados a una temperatura de $23-28^{\circ} \mathrm{C}$ con un ciclo de 12 horas de luz y 12 horas de oscuridad. Todos los animales fueron alimentados con balanceados comerciales y agua ad libitum.

\subsection{Inducción a la hiperglicemia experimental con aloxano}

El proceso de inducción a diabetes se realizó por inyección intraperitoneal de monohidrato de aloxano $(150 \mathrm{mg} / \mathrm{kg})$, siguiendo el método de Tomita ${ }^{(8)}$. Luego de dos días del tratamiento, la medición de glicemia se determinó en los animales en ayunas mediante sangre obtenida luego de corte en la cola del animal, y colocado en la tira reactiva del glucómetro. Todos los animales con glicemia superior a $180 \mathrm{mg} / \mathrm{dL}$ fueron considerados hiperglicémicos ${ }^{(9)}$.

\subsection{Grupos experimentales}

Se conformaron 4 grupos $(n=6)$, un grupo control sano y otro hiperglicémico que recibieron agua destilada, un grupo de animales normoglicémicos y otro de animales hiperglicémicos que recibieron miel $(500 \mathrm{mg} / \mathrm{kg}$, vía oral) una vez al día, en la mañana, durante tres días. La glicemia en cada grupo se determinó a las $0,1,2,4$ y $24 \mathrm{~h}$ posteriores al tratamiento.

\subsection{Análisis estadístico}

Fue utilizado el modelo de análisis estadístico ANOVA 1way. Obtenido mediante el paquete estadístico GraphPad Prism5, además de un Test de Comparación Múltiple Newman-Keuls, con un nivel de significancia estadística de valor de $p<0,05$. 


\section{RESULTADOS Y DISCUSIÓN}

Los resultados del tratamiento de los ratones por tres días por vía oral con $500 \mathrm{mg} / \mathrm{kg}$ de peso de miel de abeja indican que, en los animales sanos el consumo de miel no impacta grandemente en la glicemia, se observó un leve aumento de la glicemia una hora después de la ingesta (Figura 1). Sin embargo, no es significativamente diferente a la de la glicemia inicial (tiempo 0), y tampoco es diferente a la del grupo control que recibió agua. Adicionalmente, debe notarse aquí que el nivel de glicemia de los animales se mantuvo dentro del rango considerado como referencia, esto es entre 63 y $176 \mathrm{mg} / \mathrm{dL}^{(9)}$.

Por otro lado, en el grupo de animales con hiperglicemia inducida por el agente químico aloxano, se midió la glicemia antes y después de la ingesta de la miel de abeja. En la primera hora posterior a la administración, se verificó un aumento de la glucemia y en la segunda hora, un significativo descenso $(\mathrm{p}<0,05)$. Esto parece indicar que se produjo una estimulación de la función del páncreas dañado por el aloxano, respondiendo de la misma manera que uno sano frente a la miel de abeja (Figura 1).

En los grupos de animales que no recibieron miel, es decir un grupo normo glicémico y otro grupo hiperglicémico, tratados con agua y dieta ad libitum, se observa que la glicemia en los diferentes tiempos medidos no difiere significativamente de la glicemia inicial en cada caso. Si comparamos la glicemia entre estos dos grupos, como se espera, la diferencia es muy significativa, ya que uno de los grupos es sano y el otro recibió el aloxano y por lo tanto desarrolló hiperglicemia.

Entre los dos grupos de animales hiperglicémicos, tratados con agua y tratados con miel de abeja, respectivamente, se observó una diferencia estadísticamente muy significativa en todos los tiempos, hasta las $24 \mathrm{~h}$, esto es así porque ninguno de los dos recibió un tratamiento hipoglicemiante. Sin embargo, el resultado más resaltante en este estudio es que en el grupo de animales hiperglucémicos que recibieron la miel de abeja, luego de un incremento de la glicemia en el tiempo 1, es decir, una hora después de la ingesta, se observó posteriormente a las 2 y 4 horas un descenso significativo. Esto último parece indicar un efecto favorable de la miel de abeja en el metabolismo de la glucosa en animales hiperglicémicos (Figura 1). 
Rev. Soc. cient. Parag. 2021;26(1):91-99

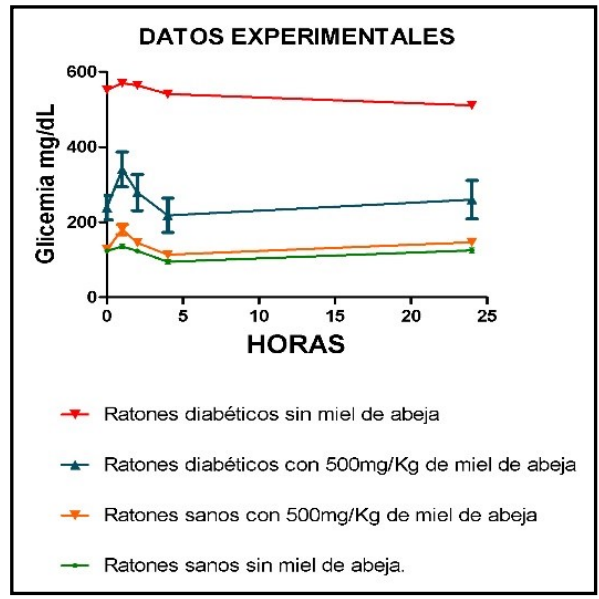

Figura 1. Evolución de la glicemia en los ratones de los diferentes grupos y efecto agudo de la miel de abeja por vía oral.

En los ratones inducidos experimentalmente a diabetes por aloxano, la deficiencia de insulina o hipoinsulinemia se desarrolla como consecuencia de la destrucción irreversible de las células $\beta$ del páncreas provocando hiperglucemia. Este efecto es dosis-dependiente, de tal manera que grandes cantidades de aloxano produce destrucción total de los islotes del páncreas, simulando una diabetes mellitus de tipo 1. Cuando se controla la dosis se puede realizar destrucciones parciales de los islotes, con lo cual se controla el nivel de hiperglucemia producida. En este trabajo se procuró y se consiguió niveles de glucemia en ayunas para animales diabéticos entre $180 \mathrm{a} 400 \mathrm{mg} / \mathrm{dl}^{(10)}$. La glicemia de los animales inducidos a hiperglicemia se mantuvo elevada por el periodo total de intervención.

La miel de abeja no afectó la glicemia de los animales sanos normoglucémicos(11), y redujo significativamente la glucemia en los hiperglicémicos en las 2 y 4 horas siguientes, similar a otros reportes

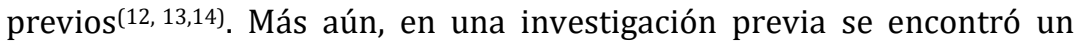
efecto sinérgico tras la administración de miel de abeja concomitante con el uso de hipoglucemiante oral en diabéticos, así como de miel de abeja y leche de camello. En ambos casos se produjo la mejoría de los niveles de glicemia en los individuos estudiados(15,16). En este estudio, se encontró que el metabolismo de la glucosa, posterior a la ingesta de 
miel en animales diabéticos parece corresponderse con un efecto ligeramente hipoglucemiante, en un modelo agudo.

Por otro lado, se debe considerar que la miel tiene un IG más bajo en comparación con muchos otros hidratos de carbono(16,17,18), ya que, en los últimos años, el control glucémico estricto pareció adquirir mayor importancia a la hora de prevenir complicaciones agudas y crónicas propias de la enfermedad y de realizar el plan alimentario para los pacientes con DM. Si bien no está claro a qué componente de la miel se debe el efecto favorable sobre el metabolismo de la glucosa, es posible que sea por la fructosa(19). La fructosa no aumenta la glicemia considerablemente y su metabolismo no requiere de la secreción de insulina(5). Un estudio anterior también informó que la fructosa estimula la secreción de insulina en páncreas aislado ${ }^{(20,21)}$. Sin embargo, entre los componentes de la miel de abeja se citan también antioxidantes, enzimas, vitaminas y minerales que podrían favorecer el metabolismo de la glucosa, la sensibilidad a la insulina y la recuperación del daño endotelial propio de la enfermedad.

La miel de abeja es un alimento muy presente en las comidas en nuestro país, es muy aceptado y empleado en gastronomía y terapéutica natural. Por lo tanto, se puede sugerir que la combinación de una dieta controlada y la adición de miel de abeja como edulcorante sustituto, podría aportar un mejor control de la glicemia en pacientes con diabetes ${ }^{(22)}$ y así poder retrasar el desarrollo y la progresión de las complicaciones diabéticas.

\section{CONCLUSIÓN}

La adición de la miel de abeja en la dieta controlada, 5 gramos de balanceado por animal y por día, de ratones con hiperglicemia experimental por aloxano, contribuye a mejorar el nivel de glicemia en este grupo de animales, por lo que podría considerarse eventualemnte el uso de miel de abeja como complemento a la dieta y alternativa al azúcar de mesa.

\section{REFERENCIAS}

1. World Health Organization. Global report on diabetes; 2016.

2. Maham L, Escott-Stump S. Nutrición y dietoterapia de Krause. Mc Graw Hill Interamericana. México; 2001. 


\section{Rev. Soc. cient. Parag. 2021;26(1):91-99}

3. Pipicelli G, Tatti P. Therapeutic properties of honey. Health. 2009;1(2):281-283.

4. Jimenez JT, Palacios M, Cañete F, Barriocanal LA, Medina U, Figueredo R, Alberti KG. Prevalence of diabetes mellitus and associated cardiovascular risk factors in an adult urban population in Paraguay. Diabetic Medicine. 1998;15(4):334-338.

5. Moore MC, Davis SN, Mann SL, Cherrington AD. (2001). Acute fructose administration improves oral glucose tolerance in adults with type 2 diabetes. Diabetes Care. 2001;24(11):1882-1887.

6. Rodríguez HGR, Méndez JD. (1994). Diabetes mellitus experimental. Ciencia Veterinaria, México. 1994;6:347-377.

7. Gil A. Tratado de nutrición. Médica Panamericana. Madrid; 2010;2:232236.

8. Tomita T. A dynamic role of liver glycogen in alloxan diabetic rats. Endocrinologia japónica. 1973;20(8):243-247.

9. Zúñiga JM, Orellana JM, Tur JA. Ciencia y tecnología del animal de laboratorio. Universidad de Alcalá de Henares y. SECAL, Madrid; 2011; 160 p.

10. Akhtar MS, Khan MS. (1989). Glycaemic responses to three different honeys given to normal and alloxan-diabetic rabbits. J Pak Med Assoc. 1989;39(4):107-13.

11. Cortés ME, Vigil P, Montenegro G. The medicinal value of honey: a review on its benefits to human health, with a special focus on its effects on glycemic regulation. Ciencia e Investigación Agraria. 2011;38(2):303317.

12. Al-Waili NS. Natural honey lowers plasma glucose, C-reactive protein, homocysteine, and blood lipids in healthy, diabetic, and hyperlipidemic subjects: comparison with dextrose and sucrose. Journal of Medicinal Food. 2004;7(1):100-107.

13. Al-Waili NS. Effects of daily consumption of honey solution on hematological indices and blood levels of minerals and enzymes in normal individuals. Journal of medicinal food. 2003;6(2):135-140.

14. Reyes MP, Morales JA, Madrigal EO. Diabetes. Tratamiento nutricional. Medicina Interna de México. 2009;25(6):454-460.

15. Erejuwa 00, Sulaiman SA, Ab Wahab MS, Sirajudeen KNS, Salleh MSM, Gurtu S. (2011). Glibenclamide or metformin combined with honey improves glycemic control in streptozotocin-induced diabetic rats. International journal of biological sciences. 2001;7(2):244-252.

16. Ahmad A, Azim MK, Mesaik MA, Khan RA. Natural Honey Modulates Physiological Glycemic Response Compared to Simulated Honey and DGlucose. Journal of food science. 2008;73(7):165-H167. 
Rev. Soc. cient. Parag. 2021;26(1):91-99

17. Agrawal OP, Pachauri A, Yadav H, Urmila J, Goswamy HM, Chapperwal A, Prasad GBKS. Subjects with impaired glucose tolerance exhibit a high degree of tolerance to honey. Journal of Medicinal Food. 2007;10(3):473478.

18. Khalil MI, Shahjahan, M., \& Absar, N. (2006). Glycemic response and glycemic index of Bangladeshi honey in type 2 diabetic patients. Malaysian. J. Pharm. Sci, 4(1), 13-19.

19. Deibert P, König D, Kloock B, Groenefeld M, Berg A. Glycaemic and insulinaemic properties of some German honey varieties. European journal of clinical nutrition. 2010;64:762-764.

20. Grodsky GM, Batts AA, Bennett LL, Vcella C, McWilliams NB, Smith D. F. Effects of carbohydrates on secretion of insulin from isolated rat pancreas. American Journal of Physiology-Legacy Content. 1963;205(4):638-644.

21. Curry DL, Curry KP, Gomez M. Fructose potentiation of insulin secretion. Endocrinology. 1972;91(6):1493-1498.

22. Ahmad A, Azim MK, Mesaik MA, Khan RA. (2008). Natural Honey Modulates Physiological Glycemic Response Compared to Simulated Honey and D-Glucose. Journal of food science. 2008;73(7):165-H167. 